\title{
Comparative study of apoptosis-detecting techniques: TUNEL, apostain, and lamin B
}

\author{
Jirina Prochazkova ${ }^{1}$, Dana Kylarova ${ }^{1}$, Pavel Vranka ${ }^{2}$, and Vaclav Lichnovsky ${ }^{1}$ \\ ${ }^{1}$ Palacky University, Olomouc and ${ }^{2}$ Masaryk University, Brno, Czech Republic
}

BioTechniques 35:528-534 (September 2003)

The mechanism of the apoptotic process is generally recognized as being highly intricate. Unfortunately, so is its detection. The concept of detection sensitivity reflects not only the ability of a particular technique to label most of the cells displaying the appropriate marker but also the possibility of labeling cells in the earliest phase of the apoptotic process. For this study, we chose three techniques, visualized by means of immunohistochemistry: terminal dUTP-transferase-mediated nick end labeling (TUNEL), apostain, and lamin B. The number of apoptotic cells detected in the observed specimens with respect to the used technique differed for the majority of cases. The lowest apoptotic indices were usually obtained by the lamin B technique, the TUNEL technique followed, and the apostain technique gave the highest values. The comparison revealed that the TUNEL and apostain techniques were positively correlated in the majority of specimens observed. The linear correlation between TUNEL/apostain and lamin B was weaker. TUNEL, apostain, and lamin B techniques, variant in principle, give different yet correlating results, which is in accordance with the assumption that they describe the same phenomenon detected at different time periods of the apoptotic process.

\section{INTRODUCTION}

A vast amount of work since the late nineteenth century has been devoted to solving the puzzle of how cells multiply. Although of equivocal importance, the question of how and why they die has been one of relatively recent concern. After this period of neglect, it was published that there is an intrinsic genetic program for scheduled removal of ineligible or redundant cells. This program is activated at a controlled time and location and was labeled programmed cell death $(1,2)$.

In literature regarding the issue of cell death, the term apoptosis (3) is frequently seen, and there is a tendency to use it as a synonym for the term programmed cell death. In actual fact, apoptosis can be taken as a type of programmed cell death, but the term apoptosis carries with it morphological connotations that do not apply to all instances of programmed cell death during development (4). Nevertheless, the majority of vertebrate developmental cell deaths occur in an apoptotic manner (5-7), and all the techniques that were used in this study are based on the classical biochemical hallmarks of apoptosis. Therefore, the term apoptosis is generally used here.

Apoptosis consists of several successive stages. Generally, most apoptotic pathways involve a sensor that detects a death-inducing signal, which triggers the death program via a signal transduction network. This leads to the activation of execution machinery (a cascade of intracellular proteases called caspases) that carries out the process of cell death. The last step is the removal of dead cells through engulfment of cell corpses by other cells, usually macrophages.

Techniques for detecting apoptosis make use of some of the many specific biochemical hallmarks of apoptosis. The most important and fairly well explored hallmark is the activation of the cascade of cystein proteases (specific for cleavage at aspartate residues) called caspases. The ability of caspase 6 to cleave lamins-especially lamin B (8)-is important for our study. Caspase 3 abolishes the inhibitor of caspase-activated DNase (ICAD/DFF-45), which enables the activation of this specific DNase (CAD/DFF-40), which is involved in the nonrandom internucleosomal degrada- tion of apoptotic DNA (9), yielding specific fragments that can be detected by the terminal dUTP-transferase-mediated nick end labeling (TUNEL) technique (10,11). Long before the beginning of DNA fragmentation, the digestion of histones and other DNA-associated proteins occurs (12). This contributes to the increased sensitivity of DNA to thermal denaturation and is the principle of the apostain technique (13). The techniques of lamin B detection, TUNEL, and apostain were chosen from a wide spectrum of methods (14) for their common characteristic of suitability to be used in combination with both paraffin-embedded tissues and cell cultures.

The aim of this study was not to describe the level of apoptosis in a given tissue, as this has already been published (15-18). Our intention was to compare the reliability, efficiency, and stability of the three techniques based on different biochemical hallmarks to give further insight into their future possible use on a broad spectrum of tissues.

\section{MATERIALS AND METHODS}

\section{Processing of Material}

For precise comparison, the following five model subjects were observed and evaluated: an anlage of embryonic limb consisting of eight morphological areas and an anlage of axial skeleton composed of three structures (both sixth through twelfth week of intrauterine development), five layers of primitive embryonic intestine (seventh through twenty-eighth week of intrauterine development), human leukemic cells HL60, and two areas of embryonic placenta in the twenty-second week of intrauterine development. Fetal and placental tissue were obtained with the full agreement of informed donors, in compliance with the Helsinky declaration and Czech legal code. HL60 cells were obtained as a courtesy of the Laboratory of Growth Regulators AS CR (Olomouc, Czech Republic).

Embryonic tissues from 25 embryos and fetuses were routinely processed-fixed in Methacarn (19) and embedded in paraffin-and $7-\mu \mathrm{m}$ thin sections were used for all detections. HL60 cells were treated for $6 \mathrm{~h}$ in 100 
$\mu \mathrm{M}$ Bohemin (20), $100 \mu \mathrm{M}$ Olomoucin $(21,22)$ (both are courtesy of the Laboratory of Growth Regulators AS CR), $100 \mu \mathrm{M}$ actinomycin D (23) (Merck, Rahway, NJ, USA), and $10 \mu \mathrm{M}$ etoposide (23) (Delta West PTI Ltd., Bentley, NSW, Australia) to induce apoptosis. Embryonic placenta was fixed in the following fixatives: $4 \%$ and $10 \%$ pure formaldehyde, $4 \%$ buffered formaldehyde, 4\% neutral formaldehyde, Bouin's fixative, Baker's fixative, methanol-phosphate-buffered saline 3: 1 (all in Reference 24), and Methacarn (19). The fixed tissue was then embedded in paraffin.

\section{TUNEL Technique}

For the detection of apoptosis, we used the following techniques. For the labeling of numerous oligonucleosomal DNA fragments of apoptotic cells by the TUNEL technique, the In Situ Cell Death Detection Kit AP (Roche Applied Science, Basel, Switzerland) with slight adjustments was used. Samples were pretreated by irradiation in a microwave oven in $0.1 \mathrm{M}$ citrate buffer, pH 6.0, for 1-15 min (according to the method of fixation of the sample). The next step consisted of the application of $30 \mu \mathrm{L}$ mixture of dUTP-transferase and fluoresceinated nucleotides (incubation for $40 \mathrm{~min}$ in $37^{\circ} \mathrm{C}$ in a humidified chamber). The same volume of antifluorescein antibody conjugated with alkaline phosphatase is applied after that (incubation for $30 \mathrm{~min}$ in $37^{\circ} \mathrm{C}$ in a humidified chamber). The procedure takes about $4 \mathrm{~h}$ and is user-friendly.

\section{Apostain Technique}

Short thermal denaturation unravels DNA in areas where DNA scaffolding was degraded by the action of caspases. Apostain is a special mouse monoclonal antibody against such ssDNA (1: 10; F7-26; Alexis Biochem, Lausen, Switzerland). Pretreatment consisted of incubation of samples dipped in tubes containing $5 \mathrm{mM} \mathrm{MgCl}$ in phosphatebuffered saline (PBS) in $99^{\circ} \mathrm{C}$ water bath for $4 \mathrm{~min}$. After $1 \mathrm{~h}$ incubation of primary antibody at $37^{\circ} \mathrm{C}$, the biotinylated secondary goat anti-mouse antibody (1: 20; Roche Applied Science) was added (for $40 \mathrm{~min}$ ). Another 40-min incubation with streptavidin-alkaline phosphate (1: 1000; Roche Applied Science) followed. The labeling of apoptotic cells by apostain takes about $7 \mathrm{~h}$, and, in comparison with common immunohistochemistry, thermal denaturation of DNA requires extra equipment.

\section{Lamin B Technique}

Loss of lamin B positivity is also a marker of apoptosis. Lamin B was detected by the standard three-step immunohistochemical assay (6-h protocol). The primary $(1: 100$; goat monoclonal antibody) and secondary (1:100; donkey anti-goat antibody) antibodies used were products of Santa Cruz Biotechnology (Santa Cruz, CA, USA); the conjugated streptavidin-alkaline phosphatase and its substrate nitro blue tetrazolium plus 5-bromo-4-chloro-3indolyl phosphate (NBT/BCIP) were produced by Roche Applied Science.

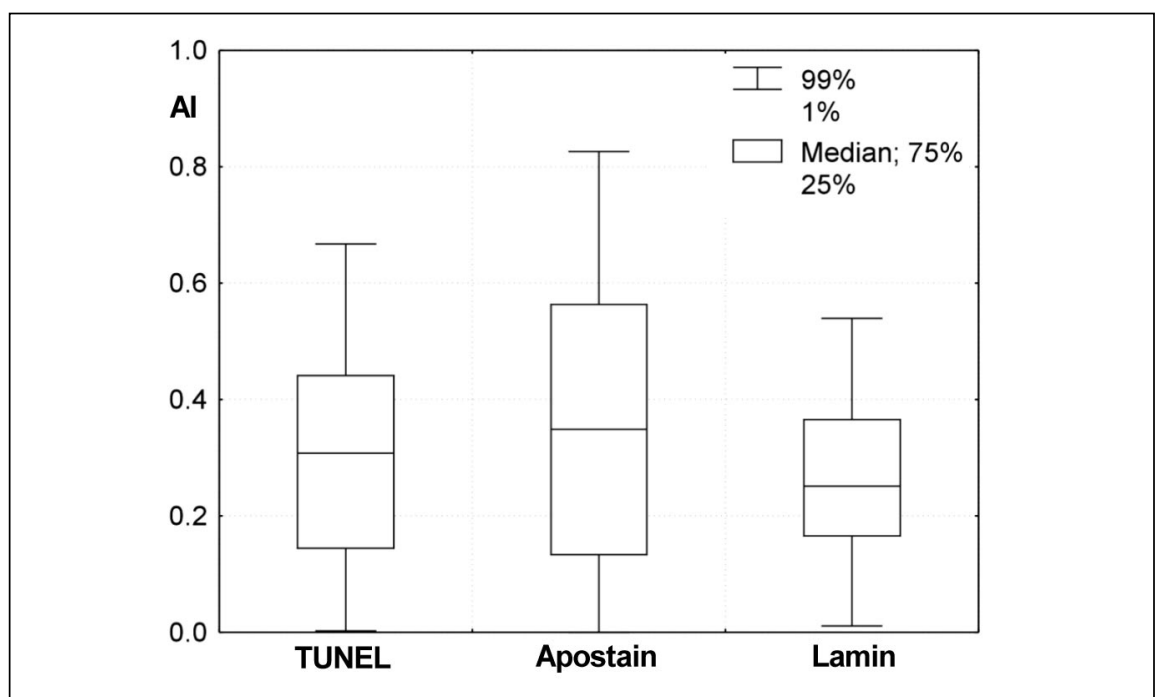

Figure 1. Box and whisker plots showing the distribution and main quartiles of all data. The location of the medians illustrates the general tendency of the apostain technique and the lamin B technique to yield the highest and lowest apoptotic indexes, respectively.

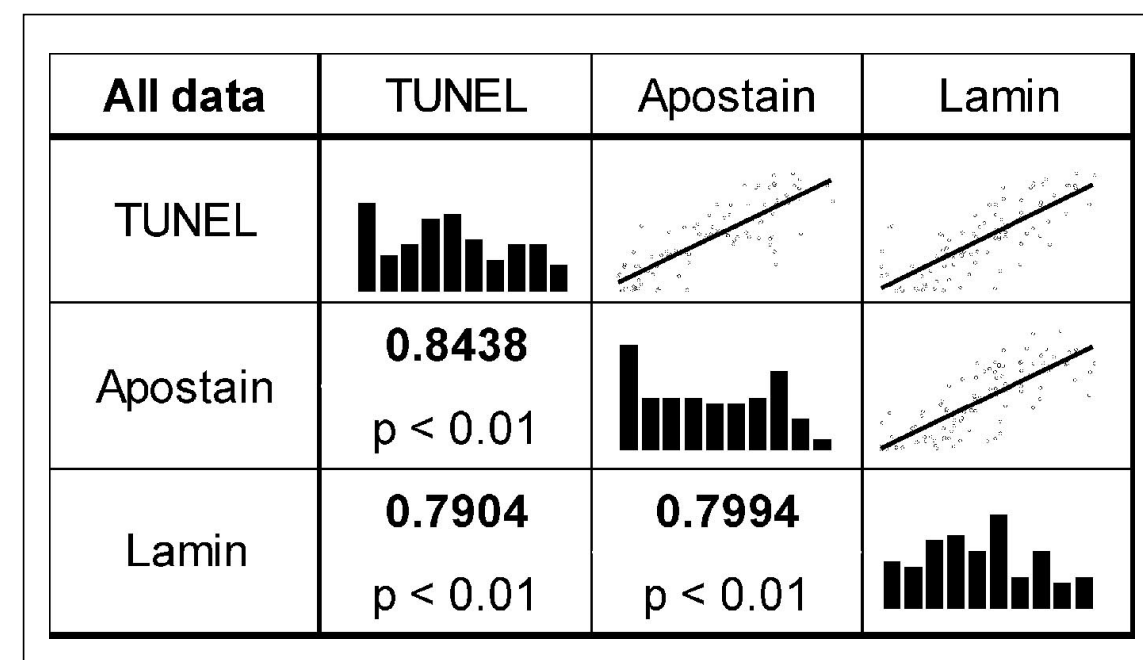

Figure 2. Correlation parameters among all the detection techniques. The correlation coefficients and their levels of significance $(P)$ are located in the left part of the table. The data histograms (bar graphs) for each of the particular techniques are in the middle [x-axis depicts apoptotic indexes (AIs) between 0 and 1 in intervals of 0.1 ; y-axis shows the total number of samples analyzed by a given technique that yielded a particular AI]. The distributions of AIs, obtained by particular combinations of detection techniques, are located in the right part of the table together with the plotted line of simple linear regression assessed by the least squares criteria. 
Purple-blue NBT/BCIP substrate was used for visualization of all immunocomplexes.

\section{Data Analysis}

The final result of any apoptosis-detecting technique is called an apoptotic index (AI). This is a ratio of apoptotic cells to the total sum of all cells. Apoptotic cells in embryonic tissues were visualized by purple-blue precipitates and quantified by means of light microscopy (Olympus Optical Co. GmbH, Hamburg, Germany) and image analysis software ACC 4.0 (Sofo, Brno, Czech Republic). For every combination of observed specimen and detection technique, the quantification was repeated six times, and the average of these six values was then subsequently used in the statistical evaluation. Leukemic HL60 cells were evaluated by flow cytometry (18), and the average value was taken from three independent measurements. Comprehensive data, including the averages of the AIs of all the samples ( $n=$ $101)$, were then processed using the statistical software Statistica for Windows (http://www.statsoftinc.com/).

The reproducibility of all the assays is high. In previously published studies, this was documented by multiple assessment of the level of apoptosis on two or three embryos of the same age or by repeating the same experiment using cell culture. All the obtained results underwent statistical evaluation.

\section{RESULTS}

\section{Selection of Evaluated Material}

The model subjects were chosen for the following features. Embryonic tissues are the physiological examples of apoptosis in the organism. They are also good models to find out whether or not the processing of tissues necessary for detection impairs the tissue morphology. HL60 cells were treated by four different apoptosis-inducing agents to assess objectively the ability of a given technique to mark apoptotic cells regardless of the agent used. Human placenta was fixed by eight different solutions to determine whether the detection techniques were influenced by fixation and whether the fixation solutions could artificially change the level of apoptosis. The results of the individual experiments involving the determination of whether or not the type of fixative used affects the level of apoptosis are not mentioned in this study. These results can be found in a previous publication (16). However, using different types of inducing agents and fixatives contributed to raising the number of samples and so widened the range of objectivity.

\section{Relations Between Techniques in Their Determination of Apoptotic Index}

The analysis of variance (ANOVA) test was used for the data variance analysis - the data proved to display balanced sorting, and a high homogeneity of variance, as tested by Bartlett. At the confidence level of less than 0.01 , the matching of data was rejected $(P=0.005656)$. This indicated that the AIs differ among all the detection techniques. Detailed analysis showed a general pattern of the quantitative level of apoptosis in association with the detection techniques in the majority of the observed objects. The level of apoptosis revealed, from lowest to highest according to technique, was the lamin B, then TUNEL, and finally the apostain technique, respectively (Figure 1). Therefore, the intention was to find out whether there was any linear dependence among pairs of techniques, and for this purpose Pearson's correlation coefficient $(r)$ was used.

\section{Correlation}

The TUNEL and apostain techniques were positively correlated in every observed organ with the exception of the placenta (limbs, $r=0.7189$; axial skeleton, $r=0.9301$; intestine, $r$ $=0.5379$; HL60, $r=0.8696$, in all the cases $P<0.0001$; placenta, $r=0.3125$, $P=0.2391)$. The lack of correlation in placental tissue is thoroughly explained in Reference 16. It is caused by the higher sensitivity of the apostain technique to the formaldehyde fixation. Although the existence of a linear correlation between TUNEL/apostain and lamin B was a bit weaker, a positive correlation between the TUNEL and the lamin B technique was appar- 
ent in certain organs. This was found to be true in the placenta $(r=0.5416$, $P<0.0001)$, limbs $(r=0.7331, P<$ $0.0001)$, and axial skeleton $(r=0.8708$, $P<0.0001)$. No relation was noted in the intestine and in HL60 cells $[r=$ -0.0351 $(P=0.8542)$ and $r=0.3802$ $(P=0.4574)$, respectively]. Nonetheless, the comprehensive correlation of all the techniques and all the data from each observed specimen revealed that there was a strong positive correlation among all three techniques (Figure 2). The Student's $t$ test also proved that the correlations were highly significant among all the techniques $(P<0.0001)$.

\section{Conversion of Results to TUNEL}

In publications regarding the issue of programmed cell death, it is apparent that the TUNEL technique is the method of choice in the majority of reports. Therefore, the aim was to find a way to convert the results obtained by the lamin B and apostain techniques to those of TUNEL. As the relation among techniques is linear, the linear regression ratio was used in the first attempt. Eventually, when testing these results using ANOVA, we did not discover any significant increase of matching $(P=$ 0.0734 ). Therefore, for purpose of conversion, we used a coefficient, which was the median of the ratios between individual techniques for each sample (TUNEL/apostain: median $=0.8594$; TUNEL/lamin B: median $=1.1215$ ) . The probability of the matching of data was then increased to 0.3401 .

There is no doubt that this trial quantification of differences among detection techniques is no more accurate than simply stating that apostain generally gives a slightly higher AI than TUNEL, etc. Therefore, the conversion coefficient should be looked upon as no more than a helpful tool for the rough estimation and comparison of AIs obtained by one of these techniques.

\section{DISCUSSION}

Techniques for the detection of apoptosis are numerous and variant-they detect specific hallmarks of apoptosis and incorporate several different methods as are flow cytometry, immunohisto(cyto)chemistry, ELISA staining with charged dyes, electrophoresis, etc. Among all these detection techniques, TUNEL is the most widely used. Initially, immediately after the development of the technique, TUNEL was highly respected as the most reliable and specific way to label apoptotic cells. Then, the danger of false-positively stained necrotic cells challenged its reliability, but now TUNEL has been gradually gaining more respect. The main advantage of TUNEL is its versatility-it allows the comparison of fixed and live material, cells and tissues, even animals and plants. Therefore, it is a prime candidate for standardization. Our conversion coefficients could be helpful not only when approximately comparing results produced in other laboratories but also when comparing those from one technique to those from two others. They also conserve precious embryonic material, since only one technique is performed and results can then be approximately standardized.

As each of the apoptosis detecting techniques has a weak point, more than one detection technique is usually used for precise assessment of the apoptosis level in the observed subject (25). Usually, a combination of an immunohistochemical technique (mostly TUNEL) and ELISA or flow cytometry is used. However, for paraffin-embedded material, immunohistochemistry is the only available approach. Therefore, this study gives the comparison among assays suitable for immunohistochemistry. It is no surprise that the TUNEL technique is so ubiquitous-it is timesaving and gives the most coherent results. On the other hand, it involves an empirically determined step of pretreatment, which cannot be the same for all samples. Even if the procedural steps involved in the apostain and the lamin B techniques are more constant, the apostain technique is more time-consuming and less userfriendly. The greatest weakness of the lamin B technique is that the apoptotic cells to be detected are the ones that are not stained at all and are therefore more difficult to find in densely stained tissue. That handicaps this quite robust detection technique, which is actually affected less by the method of tissue fixation than the other two techniques (16).
The stability of studied techniques involves their tolerance to (i) slightly changing conditions, (ii) the method of pretreatment, and (iii) the ability to avoid false-positive or false-negative results. Lamin B is the most stable technique regarding the first two parameters, but it could give false-positive results, as during mitosis lamins are degraded as well. Detection by the apostain technique, on the other hand, is hardly affected by the method of tissue fixation. The critical point of TUNEL technique is the nonuniform pretreatment procedure and its ability to stain even late necrotic cells. Therefore, we cannot simply state what is the most stable technique-this is possible to determine only with reference to the proliferation status, the occurrence of necrosis, and the fixation fluid of a given tissue that is used.

To recapitulate, these techniques give different but strongly correlating results because they describe the same phenomenon detected at different phases in the course of the apoptotic process. The degradation of protein scaffolding of DNA occurs in the early part of the apoptotic process, whereas fragmentation of DNA and disintegration of lamin $\mathrm{B}$ in the nuclear membrane are later events influenced by downstream caspases. This reality partially explains the generally higher amount of cells stained using the apostain technique. On the other hand, the low numbers of detected lamin B-negative cells are most probably caused mainly by their lower visibility. Moreover, the results obtained by lamin B correlate less; therefore, in further experimentation, priority will be given to the preferred TUNEL and apostain techniques.

\section{ACKNOWLEDGMENTS}

This study was supported by the Grant Agency of the Czech Republic grant no. 304/01/0485, and by Ministry of Education, Youth, and Sports grant no. 151100001. It also would not have been possible without the help of the Laboratory of Molecular Pathology, Palacky University and the Institute of Experimental Botany, Academy of Sciences, Czech Republic (both in Olomouc, Czech Republic). 


\section{REFERENCES}

1.Lockshin, R.A. and C.M. Williams. 1964. Programmed cell death I. Endocrine potentiation of the breakdown of the intersegmental muscles of silkmoths. J. Insect Physiol. 10: 643-649.

2.Lockshin, R.A. and C.M. Williams. 1965 Programmed cell death II. Cytology of degeneration in the intersegmental muscles of the pernyi silkmoth. J. Insect Physiol. 11: 123-133.

3.Kerr, J.F.R., A.H. Wyllie, and A.R. Currie. 1972. Apoptosis: a basic biological phenomenon with wide-ranging implications in tissue kinetics. Br. J. Cancer 26:239-257.

4.Majno, G. and I. Joris. 1995. Apoptosis, oncosis, and necrosis-an overview of cell death. Am. J. Pathol. 146:3-15.

5.Franz, T.A. and S.H. Kidson. 1997. Mapping of interdigital apoptosis in the chick and duck hindlimb. S. Afr. J. Sci. 93:85-90.

6.Smith, S.M. 1997. Alcohol-induced cell death in the embryo. Alcohol Health Res. World 21: 287-303.

7.Lichnovsky, V., Z. Kolar, P. Murray, A. Hlobilkova, D. Cernochova, E. Pospisilova, B. Vojtesek, and R. Nenutil. 1998. Differences in $\mathrm{p} 53$ and $\mathrm{Bcl}-2$ expression in relation to cell proliferation during the development of hu- man embryos. BMJ Mol. Pathol. 51:131-137.

8.Buendia, B., A. Santa-Maria, and J.C. Courvalin. 1999. Caspase-dependent proteolysis of integral and peripheral proteins of nuclear membranes and nuclear pore complex proteins during apoptosis. J. Cell Sci. 112: 743-1753.

9.Inohara, N., T. Koseki, S. Chen, M.A. Benedict, and G. Nunez. 1999. Identification of regulatory and catalytic domains in the apoptosis nuclease DFF40/CAD. J. Biol. Chem. 274:270-274.

10.Gavrieli, Y., Y. Sherman, A. Ben-Sasson. 1992. Identification of programmed cell death in situ via specific labeling of nuclear DNA fragmentation. J. Cell Biol. 119:493-501.

11.Gorczyca, W., J. Gong, and Z. Darzynkiewicz. 1992. DNA strand breaks occurring during apoptosis: their early in situ detection by the terminal deoxynucleotidyl transferase and nick translation assays and prevention by serine protease inhibitors. Int. J. Oncol. 1:639-648.

12.Hotz, M.A., F. Traganos, and Z. Darzynkiewicz. 1992. Changes in nuclear chromatin related to apoptosis or necrosis induced by the DNA topoisomerase II inhibitor fostriecin in MOLT-4 and HL-60 cells are revealed by altered DNA sensitivity to denaturation. Exp. Cell Res. 201:184-191.
13.Frankfurt, O.S., J.A. Robb, E.V. Sugarbaker, and L. Villa. 1997. Apoptosis in breas carcinoma detected with monoclonal antibody to single-stranded DNA: relation to bcl-2 expression, hormone receptors, and lymph node metastases. Clin. Cancer Res. 3:465-471.

14.Darzynkiewicz, Z. and F. Traganos. 1998 Measurement of apoptosis. Adv. Biochem. Eng. Biotechnol. 62:33-73.

15.Lichnovsky, V., J. Prochazkova, B. Erdosova, D. Nepozitkova, and Z. Kolar. 1999. The role of the apoptosis and the genes regulating apoptosis in early differentiation of human embryo. Gen. Physiol. Biophys. 18(Suppl 1):96-99.

16.Prochazkova, J., B. Erdosova, M. Pecuchova, and P. Vranka. 2000. Effect of a fixation and detection techniques to the assessment of apoptosis. Acta Univ. Palacki Olomouc Fac. Med. Biomed. Papers 144:87-88

17.Kylarova, D., J. Prochazkova, J. Madarova, and J. Bartos. 2001. Comparison of methods TUNEL, M30 and annexin V by flow cytometry. Histochem. J. 33:489.

18.Kylarova, D., J. Prochazkova, J. Madarova, J. Bartos, and V. Lichnovsky. 2002. Comparison of the TUNEL, lamin B and annexin $\mathrm{V}$ methods for the detection of apoptosis by flow cytometry. Acta Histochemica 104: 367-370. 
19.Puchtler, H., F.S. Waldrop, S.N. Meloan, M.S. Terry, and H.M. Conner. 1970. Methacarn (methanol-Carnoy) fixation. Practical and theoretical considerations. Histochemie 21:97-116.

20.Kovarova, H., M. Hajduch, G. Korinkova, P. Halada, S. Krupickova, A. Gouldsworthy, N. Zhelev, and M. Strnad. 2000. Proteomics approach in classifying the basic of the anticancer activity of the new olomoucine-derived synthetic cyclin-dependent kinase inhibitor, bohemine. Electrophoresis 21:3757-3764.

21.Hajduch, M., Z. Kolar, R. Novotny, J. Hanus, V. Mihal, A. Hlobilkova, V. Noskova, and M. Strnad. 1997. Induction of apoptosis and regression of spontaneous dog melanoma following in vivo application of synthetic cyclin-dependent kinase inhibitor olomoucine. Anticancer Drugs 8:1007-1013.

22.Hajduch, M., L. Havlicek, J. Vesely, R. Novotny, V. Mihal, and M. Strnad. 1999. Synthetic cyclin dependent kinase inhibitors: new generation of potent anti-cancer drugs. Adv. Exp. Med. Biol. 457:341-353.

23.Verhaegen, S., A.J. McGowan, A.R. Brophy, R.S. Fernandes, and T.G. Cotter. 1995. Inhibition of apoptosis by antioxidants in the human HL-60 leukemia cell line. Biochem. Pharmacol. 50:1021-1029.

24.Junqueira, L.C., J. Carneiro, and R.O. Kelley. 1992. Basic Histology, 7th ed. PrenticeHall International, East Norwalk, CT.

25.McCloskey, T.W., S. Chavan, S.M. Lakshmi Tamma, and S. Pahwa. 1998. Comparison of seven quantitative assays to assess lymphocyte cell death during HIV infection: measurement of induced apoptosis in anti-Fas-treated Jurkat cells and spontaneous apoptosis in peripheral blood mononuclear cells from children infected with HIV. AIDS Res. Hum. Retroviruses 14:413-1422.

Received 19 February 2003; accepted 21 May 2003.

Address correspondence to Jirina Prochazkova, Department of Histology and Embryology, Medical Faculty of Palacky University, 77515 Olomouc, Czech Republic.e-mail:jipro@tunw.upol.cz.

\title{
Purification of Mycobacterium leprae RNA for gene expression analysis from leprosy biopsy specimens
}

\author{
Diana L. Williams, Sandra Oby-Robinson, Tana L. Pittman, \\ and David M. Scollard \\ National Hansen's Disease Programs at SVM, LSU, Baton Rouge, LA, USA
}

BioTechniques 35:534-541 (September 2003)

Gene expression analysis in Mycobacterium leprae, an obligate intracellular pathogen and the etiologic agent of leprosy, has been hampered by the lack of an efficient method to purify RNA from leprosy lesions. Therefore to date, transcripts for only a few genes have been identified. We report the use of a single-tube homogenization/RNA extraction method that produces enough RNA to study the expression of 30 genes from a single skin biopsy specimen of a multibacillary leprosy patient and demonstrate that RNA can be purified after fixation of biopsies in $70 \%$ ethanol for up to a year. This represents a major advancement in the ability to study M. leprae gene expression directly from biopsy material and should help to define genes that are associated with intracellular survival of this human pathogen.

\section{INTRODUCTION}

Mycobacterium leprae, the etiologic agent of leprosy, affects the skin, eyes, and mucosa of the upper respiratory tract and is unique among bacterial pathogens in its ability to invade the peripheral nervous system (1). Approximately 3 million leprosy patients in the world are physically disabled as a result of damage to peripheral nerves and the attendant sensorimotor loss. One-fourth of the 700,000 newly diagnosed patients each year (2) will suffer from irreversible nerve damage and are at risk of developing classic hand and foot deformities and associated disabilities as a long-term consequence of infection with M. leprae (3).

Fundamental to understanding the pathogenicity of $M$. leprae is to define the composite phenotype of the bacillus and to determine gene expression related to intracellular survival and virulence. A major obstacle impeding progress in this area has been our inability to culture $M$. leprae in vitro. Lacking sufficient quantities of viable $M$. leprae from biopsy material of leprosy lesions from which to study the biochemical basis of phenotype and genotype, most findings in this area have been of limited scope. In addition, gene expression analysis of $M$. leprae has been greatly hampered by the lack of a suitable method to purify RNA from infected tissues in sufficient quantities to analyze a large number of genes from a single biopsy. To date, transcripts for only a few $M$. leprae genes have been identified $(4,5)$. Therefore, there is a general lack of understanding of which genes are required for intracellular survival and virulence.

A major reason for the inability to purify $M$. leprae RNA from clinical specimens is that $M$. leprae possesses a complex mycobacterial surface structure consisting of a Gram-positive-like cell wall surrounded by a waxy, lipid, mycolic acid- and phenolic glycolipidcontaining envelope (6). This dense coat makes lysis of $M$. leprae for subsequent purification of RNA extremely difficult. Therefore, conventional techniques for purification of mycobacterial RNA for gene expression analysis have not been successfully applied to generate sufficient quantities of $M$. leprae mRNA, even when large numbers of bacteria from infected mouse foot pads have been extracted $(4,5)$. To complicate this issue, only low numbers of bacilli $\left(<10^{9}\right)$ are recovered from skin biopsies of leprosy lesions (7). Recently, we have described a method to purify RNA from purified bacteria, which employs a single-tube homogenization/RNA 